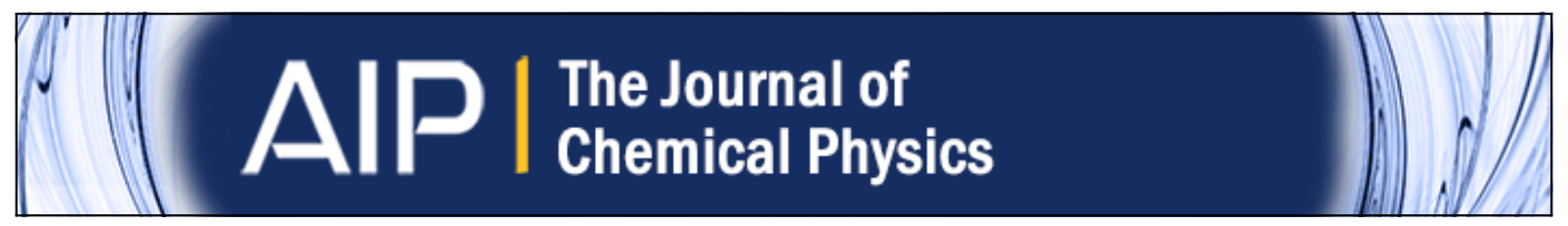

Effect of high hydrostatic pressure on the dielectric relaxation in a non-crystallizable monohydroxy alcohol in its supercooled liquid and glassy states

S. Pawlus, M. Paluch, M. Nagaraj, and J. K. Vij

Citation: The Journal of Chemical Physics 135, 084507 (2011); doi: 10.1063/1.3626027

View online: http://dx.doi.org/10.1063/1.3626027

View Table of Contents: http://scitation.aip.org/content/aip/journal/jcp/135/8?ver=pdfcov

Published by the AIP Publishing

AfP Re-register for Table of Content Alerts 


\title{
Effect of high hydrostatic pressure on the dielectric relaxation in a non-crystallizable monohydroxy alcohol in its supercooled liquid and glassy states
}

\author{
S. Pawlus, ${ }^{1}$ M. Paluch, ${ }^{1}$ M. Nagaraj, ${ }^{2}$ and J. K. Vij ${ }^{2, a)}$ \\ ${ }^{1}$ Institute of Physics, University of Silesia, Katowice, Uniwersytecka 4, 40-007, Poland \\ ${ }^{2}$ Laboratory of Advanced Materials, Department of Electronic and Electrical Engineering, \\ Trinity College, University of Dublin, Dublin 2, Ireland
}

(Received 27 May 2011; accepted 26 July 2011; published online 25 August 2011)

\begin{abstract}
The complex relative permittivity of a non-crystallizable secondary alcohol, 5-methyl-2-hexanol, is measured over a wide range of temperatures and pressures up to $1750 \mathrm{MPa}(17.5 \mathrm{kbar})$. The data at atmospheric pressure $(P=0.101 \mathrm{MPa})$ are analyzed in terms of three processes, and the results are in complete agreement with that of O. E. Kalinovskaya and J. K. Vij [J. Chem. Phys. 112, 3262 (2000)]. Process I is of the Debye type and process II is of the Davidson-Cole type, whereas process III is identified as the Johari-Goldstein relaxation process. For pressures of $\sim 500 \mathrm{MPa}$ and higher, processes I and II are seen to merge into each other to form a single dominant process which unambiguously cannot be resolved into more than one process. The dielectric relaxation strength of process I decreases slightly initially with pressure and when the two processes have merged at elevated pressures, the total relaxation strength increases with increase in pressure. Process III is better resolvable at higher pressures especially above $T_{g}$ in the supercooled liquid state for the reason that the separation in the time scales between the dominant and the JG relaxation process increases at elevated pressures. Surprisingly we find a change in the slope in the plot of $\log \tau_{J G}$ vs. 1/T for $P$ $=1750 \mathrm{MPa}$. The results for the relaxation time of alcohols are compared with the Kirkwood correlation factor, $g$, and it is found that higher is the $g$, lower is the relaxation time for process I, and it is more of the Debye type. On a reduction in $g$ brought about by an increase in pressure at lower temperatures, the dominant process becomes non-Debye though extensive hydrogen bonding is still present. The dielectric strength of the merged processes increases with increase in pressure. The values of the steepness index, $m=\left|\mathrm{d} \log \tau / \mathrm{d}\left(T_{g} / T\right)\right|_{T=T g}$ for processes I and II are different for $P$ $=0.1 \mathrm{MPa}$. However the value of $m$, for the composite process, which is a merger of processes I and II, for $P=1750 \mathrm{MPa}$ is almost the same for process II at $P=0.1 \mathrm{MPa}$. From the results of the activation volume, activation enthalpy, and a comparison of the relaxation times with the $g$ factor, we conclude that both processes I and II are significantly affected by hydrogen bonding and both contribute to the structural relaxation. (C) 2011 American Institute of Physics. [doi:10.1063/1.3626027]
\end{abstract}

\section{INTRODUCTION}

The effect of pressure on the dielectric relaxation behavior of liquids has been a subject of enormous interest since the 1960s. ${ }^{1-13}$ Work at the time was hampered by the long and tedious manual procedures required to make dielectric measurements for different pressures at different temperatures. More recently, however automated data collection techniques as well as the data analysing techniques ${ }^{14,15}$ have made such studies more revealing of the effects observed at pressures especially exceeding $500 \mathrm{MPa}(5 \mathrm{kbar})$. Considerable attention has been focused on to the hydrogen-bonded alcohols. These studies were initially made for the reason that dynamics in alcohols is much slower and simpler ${ }^{1,16,17}$ and exhibited dispersion at frequencies where measurements could have easily been made. Furthermore these gave a basis for the exploration of the phenomenon of dynamics in liquids under hydrostatic pressure for different temperatures with pressure as an addi-

\footnotetext{
a) Author to whom correspondence should be addressed. Electronic mail: jvij@tcd.ie.
}

tional thermodynamic variable to temperature. However recent studies using high hydrostatic pressure ${ }^{18-20}$ are motivated mainly by two reasons: (i) to resolve a controversy that exists as to whether the predominant process observed in some of the monohydroxy alcohols, which is Debye-like, contribute to the structural relaxation. In this context, the structural relaxation refers to the manner in which the system returns to equilibrium after the field is removed, and whether motions responsible for viscosity and dc conductivity contribute to it, and (ii) to find as to how the effects due to hydrogen-bonding are manifested in the physical properties of biological systems so as to advancing their understanding. Water is the most simple and ubiquitous liquid and it plays the most important role in biology. Due to the formation of an almost well defined unit of co-ordinated structure through hydrogen bonding in the liquid state, it cannot easily be supercooled due to the onset of crystallization at the freezing temperature. A detailed discussion of the first motivation will be made in Sec. IV B.

It has been found that while an initial application of pressure promotes hydrogen bond formation in alcohols, 
application of further higher pressure deforms or weakens these bonds, and hence the population of hydrogenbonded structural motifs is reduced. Since hydrogen bonding effects the orientational correlation of dipoles through short-range dipolar interactions given by Onsager-KirkwoodFröhlich theory, ${ }^{21}$ it changes the equilibrium dielectric permittivity due to both changes in the density and an alteration in the structure but except for cases where it leads to ring dimer formation. ${ }^{12,22,23}$ Pressure in general raises the static dielectric permittivity. ${ }^{5,12}$ Pressure has pronounced effects on the dielectric relaxation time especially at lower temperatures. ${ }^{5,13}$ For experimental investigations up to $400 \mathrm{MPa}$ (4 kbar) and for frequencies in the range $50 \mathrm{mHz}$ to $0.5 \mathrm{MHz}$ and temperatures down to $215 \mathrm{~K}$, Johari and Dannhauser made for the first time a detailed investigation ${ }^{5}$ of five isomeric octanols and found that the predominant dispersion is well described by the Debye equation in only some of these octanols. However they observed higher frequency dispersions and departures from the Debye behavior in four out of the five compounds. They were easily able to resolve a number of dispersions in three out of the five isomeric octanols. For example, in 6-methyl-3-heptanol, three dispersions at a temperature of $218 \mathrm{~K}$ were reported and consequently they determined three relaxation times. Furthermore they found that the amplitude of process III increased with pressure and stated it was "a surprising observation" but at the time they did not ascribe any cause to this process. This is now known as the Johari-Goldstein $(\mathrm{JG})$ relaxation discovered one year later to these early indicative observations made in a different context. ${ }^{24}$ Johari and Dannhauser $^{5}$ also found that process II especially at lower temperatures was of the Davidson-Cole type. ${ }^{25}$ This work was extended by Vij et al. $^{12,13}$ to an investigation of four heptanol isomers (1heptanol, 3-heptanol, 3-methyl-2-hexanol, and 2-methyl-2hexanol) who investigated these liquids in the frequency range of $20 \mathrm{~Hz}$ to $6 \mathrm{MHz}$ and temperatures from $373 \mathrm{~K}$ to $243 \mathrm{~K}$. Since their low frequency measurements were limited to $20 \mathrm{~Hz}$ only, they did not observe the non-Arrhenius nature of the dominant process at the lowest temperatures. The nature of the dominant process was of the Debye-type in 1-heptanol. However a slight departure from the Debye process was observed in 3-heptanol and the Davidson-Cole ${ }^{25}$ type behavior was found in the other two compounds: 3-methyl-2-hexanol and 2-methyl-2-hexanol. The results revealed that DavidsonCole parameter $\beta$ for the dominant process decreased or the distribution of relaxation times increased with an increase in pressure and the values of the parameter $\beta$, so calculated from the fits, were tabulated.

Several monohydroxy alcohols and amides are known to exhibit three relaxation times in their dielectric spectra. ${ }^{26-28}$ The lowest frequency process in these systems is designated as process I. This is almost of the Debye-type. The next higher frequency, designated as process II, shows broader distribution of relaxation times and is usually of the DavidsonCole $^{25}$ type, and the highest frequency process is designated as process III. Though weak in dielectric strength, it has a much wider distribution of relaxation times but with almost Arrhenius temperature dependence of the relaxation time and is identified as the JG relaxation. ${ }^{24}$ One of the monohydroxyl alcohols 2-ethyl-1-hexanol has been studied extensively in the literature mainly with a view to resolving as to which of the two processes I or II, is responsible for the structural relaxation. ${ }^{18-20,29-31}$ In particular, dielectric features have been compared with the observations made from calorimetry, ${ }^{29}$ mechanical spectroscopy, ${ }^{29}$ and recently NMR. ${ }^{31}$ The effects of high pressure on dielectric properties have been investigated by three groups. ${ }^{18-20}$ A comparison of these results with those found using alternative complementary techniques has led to a controversial interpretation. Fragiadakis et al. ${ }^{18}$ have given a brief and updated review of this controversy.

This relaxation in its simplest form pertains to the dynamics that ceases in the experimental time span of an observer, and the relaxation time is conventionally fixed corresponding to a frequency of $10^{-4} \mathrm{~Hz}$ for which $\varepsilon^{\prime \prime}$ is maximum as the system approaches the glass transition temperature (according to the definition used in some of our previous works). ${ }^{32-34}$ In differential scanning calorimetry (DSC) the time is fixed at $100 \mathrm{~s}$, which many researchers in dielectric spectroscopy have also adopted. In fact the structural relaxation may correspond to almost all the fluctuations that cease at the glass transition temperature. These exclude those corresponding to the JG mode which still persist in the glassy state; the motions corresponding to JG mode are localized. ${ }^{24}$ Hence if the state of the liquid at a given temperature were to explore a large number of configurations of equal energy, then these configurations would increase a liquid's configurational entropy $S_{c}$. (Note that $S_{c}$ is determined by using the integral of the excess specific heat $\Delta C_{P}$, of a liquid over the crystal phase value at constant pressure, $S_{c}=\int_{T_{0}}^{T}\left(\Delta C_{P} / T\right) d T ; T_{0}$ is the temperature where $S_{c} \rightarrow \mathrm{S}_{\mathrm{c}(\text { crystal phase) }}$ ). The state point of a liquid explores configurations of different energies at different temperatures but at a given temperature only configurations of the same energy determine $S_{c}$. There will thus be a temperature-dependent configurational contribution to entropy and it is observed as a change in $\Delta C_{P}$ with $T$. If the frequency of the small-amplitude heat flux (perturbation) to the sample is changed, the real and imaginary parts of the dynamic specific heat depend on frequency, as is observed in ultraviscous liquids at temperatures close to $T_{g}$. (This dependence determines how the heat flux is absorbed into different modes of molecular motions.) All structural fluctuations amongst configuration of equal energy are included in the dynamic behavior and reflected in the dynamical specific heat of an equilibrium liquid.

From measurements on 5-methyl-2-hexanol, Tombari et $a l .{ }^{38}$ have found out that the calorimetric relaxation time is different from the dielectric relaxation time of both processes and the distribution of relaxation times found from such studies is also not the same as found from dielectric spectroscopy. Calorimetric relaxation requires configurational fluctuations which do not require a change in the dipole vector whereas the dielectric relaxation requires that the dipole vector lag behind the electric field vector. Also, the distribution of relaxation times for the calorimetric and the mechanical relaxation is found not to be the same as seen by dielectric dispersion. Calorimetry as already implied explores all molecular degrees of freedom, whereas the mechanical spectroscopy explores 
the elastic deformations following the application of the mechanical field, and the results are also not specifically related to the molecular behavior either. Structural fluctuations may not correspond to the H-bond fluctuations especially when the reference molecule reforms a bond with the same neighboring molecule. It should therefore not be a surprise to us when the distribution of relaxation times observed by one technique differs from that given by the other and the determined values of the kinetic glass transition temperature $T_{g}$ differ somewhat from each other. It has already been shown that $T_{g}$ determined using dielectric spectroscopy for process I is higher than for process II by a temperature of $\sim 1-3 \mathrm{~K}$ (Ref. 35) (see Table I, last column in Ref. 35). This is valid for both 5-methyl-2-hexanol ${ }^{32}$ and 1-propanol ${ }^{36}$ in their neat forms as well as in their solutions of $0.5 \%$ and $1 \%$ of salt $\left(\mathrm{LiClO}_{4}\right) .{ }^{35-37}$ This salt is known to partially break the hydrogen bonds.

In this paper, we report the dielectric permittivity, $\varepsilon^{\prime}$, and the dielectric loss, $\varepsilon^{\prime \prime}$, spectra of a neat monohydroxy alcohol 5-methyl-2-hexanol up to a high pressure of $1750 \mathrm{MPa}$ (17.5 kbar) for different temperatures. The dielectric spectroscopy of 5-methyl-2-hexanol in neat liquid form, ${ }^{32}$ in solutions of both non-polar ${ }^{33}$ and polar solvents ${ }^{34}$ and by the addition of $\mathrm{LiClO}_{4}$ salt, ${ }^{35}$ have already been reported. The limitations of the Debye-Stokes-Einstein equation are already discussed. ${ }^{35,37}$ The DSC for a range of temperatures has also been investigated. ${ }^{38}$ The various findings made so far on 5-methyl-2-hexanol are summarized in a recent paper. ${ }^{33}$

\section{EXPERIMENTAL METHODS}

5-Methyl-2-hexanol of analytical grade of purity $\geq 98 \%$, purchased from Sigma Aldrich, was distilled under vacuum. The distilled middle fraction was analyzed using NMR and the water content of $<0.05 \%$ was detected, and small traces of $\leq 0.5 \%$ of the other isomers of heptanol were found. In the previous works, ${ }^{32-35,37}$ purum grade of 5-methyl-2-hexanol of purity $>99.8 \%$ purchased from Fluka A. G. Switzerland was used. However, no difference in the results of the dielectric permittivity for the two samples of 5-methyl-2-hexanol, obtained under the same experimental conditions, was found. The dielectric measurements were carried out using Novocontrol Alpha-A Analyzer and ZG4 dielectric interface in the frequency range between $50 \mathrm{mHz}$ and $3 \mathrm{MHz}$. The results of complex dielectric permittivity at the atmospheric pressure of $\sim 0.1 \mathrm{MPa}$ and for the various selected temperatures investigated agreed completely with those made previously by Kalinovskaya and $\mathrm{Vij} .{ }^{32}$ The design of the high pressure technique used herein is very similar to that used by Johari and Whalley. ${ }^{9}$ The experimental pressure setup was constructed by Unipress Ltd., Poland where a homemade parallel plate capacitor, with two standing semicircular flat-faced steel electrodes, bonded together by two teflon rings one on either ends, could be inserted in the bore of the pressure vessel of diameter $10 \mathrm{~mm}$. A spacing of $0.5 \mathrm{~mm}$ provided by teflon spacers inserted in between the two flat faced electrodes of the cell was obtained. The electrical connections were made to the bottom of the each of the two electrodes. The pressure was exerted on the sample by a steel piston. The sample under experimental investigation was in contact with only stainless steel and teflon. During high pressure measurements, temperature was controlled to within $0.5 \mathrm{~K}$ using a Tenney thermostatic system. The accuracy in temperature at the atmospheric pressure is maintained for several hours to within $\pm 0.1 \mathrm{~K}$, as required by the dielectric measurements at $\mathrm{mHz}$ frequencies.

\section{EXPERIMENTAL OBSERVATIONS AND DATA ANALYSIS}

The $\varepsilon^{\prime}$ and $\varepsilon^{\prime \prime}$ spectra of 5-methyl-2-hexanol are recorded over a temperature range of $340 \mathrm{~K}$ and $209 \mathrm{~K}$ and for pressures up to $1750 \mathrm{MPa}$. For atmospheric pressure, the temperature range investigated is much wider and the accuracy and the stability of the temperatures are much higher. Figures 1(a) and 1 (b) present the $\varepsilon^{\prime}$ and $\varepsilon^{\prime \prime}$ data at a temperature of $293 \mathrm{~K}$ for selected pressures. Figures 2(a) and 2(b) show the $\varepsilon^{\prime}$ and $\varepsilon^{\prime \prime}$ data for pressures of $512 \mathrm{MPa}$ and selected temperatures in the range $201 \mathrm{~K}$ to $293 \mathrm{~K}$. Figures 3(a) and 3(b) show the $\varepsilon^{\prime}$ and $\varepsilon^{\prime \prime}$ data at a pressure of $1750 \mathrm{MPa}$ for selected temperatures in the range $209 \mathrm{~K}$ to $339 \mathrm{~K}$. The data were recorded by selecting the temperature and by increasing the pressure. At several temperatures, the sample at a higher pressure was left overnight to find if any changes in the dielectric properties with time were observed. This was not the case for the investigated sample. This also implied that the 5-methyl-2-hexanol did not freeze with time under high pressure.

The $\varepsilon^{\prime}$ and $\varepsilon^{\prime \prime}$ data are analyzed using the HavriliakNegami ${ }^{39}$ equation for the complex permittivity given below

$$
\varepsilon^{*}(\omega)=\varepsilon^{\prime}-j \varepsilon^{\prime \prime}=\varepsilon_{\infty}+\sum_{i=1}^{I I I} \frac{\Delta \varepsilon_{i}}{\left(\left(1+\left(j w \tau_{H N i}\right)^{\alpha_{i}}\right)^{\beta_{i}}\right.}-\frac{j \sigma_{d c}}{\varepsilon_{0} \omega},
$$

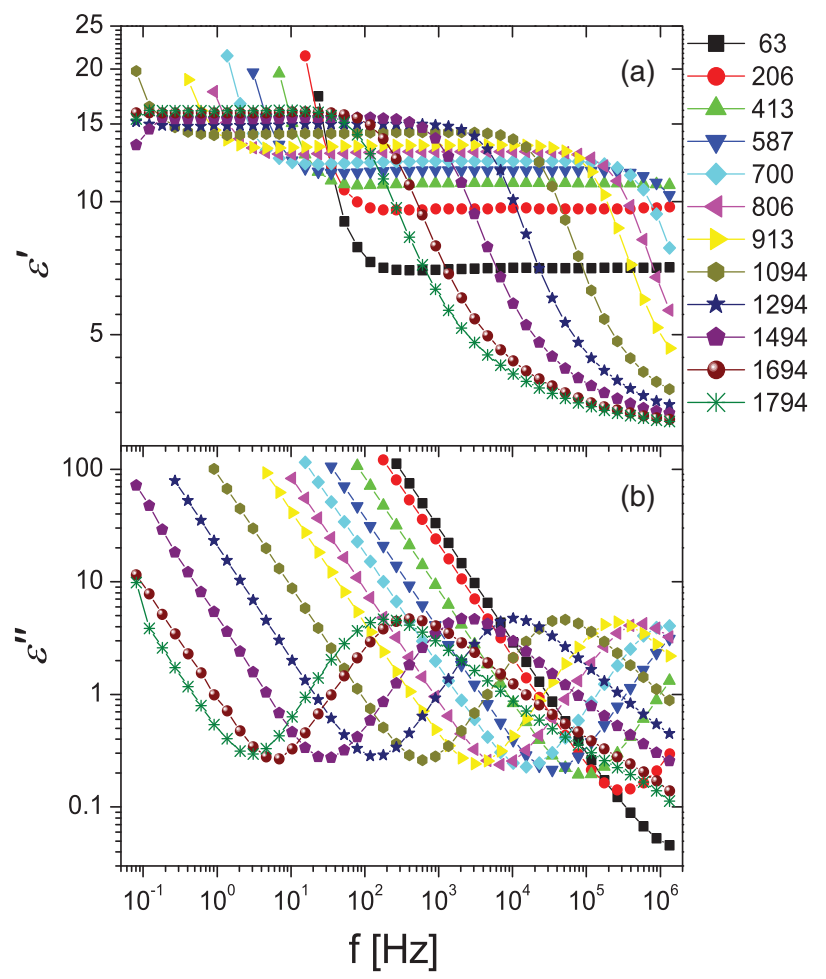

FIG. 1. (a) The dielectric permittivity, $\varepsilon^{\prime}$, and (b) $\varepsilon^{\prime \prime}$ spectra of 5-methy-2hexanol at a temperature of $293 \mathrm{~K}$ at selected pressures varying from $0.1 \mathrm{MPa}$ to $1750 \mathrm{MPa}$. The pressure values given are in MPa. 


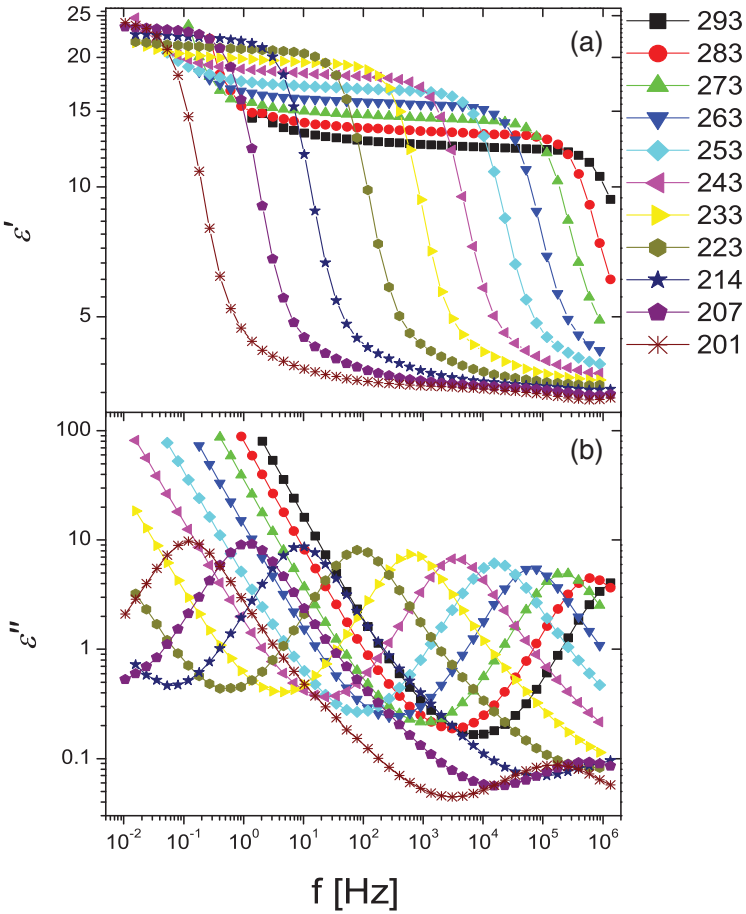

FIG. 2. (a) The dielectric permittivity, $\varepsilon^{\prime}$, and (b) $\varepsilon^{\prime \prime}$ spectra $P=512 \mathrm{MPa}$ for different temperatures. The temperatures given are in $\mathrm{K}$.

where $\omega=2 \pi f$ ( $f$ being the frequency in given $\mathrm{Hz}$ ), and $\varepsilon_{0}$ is the permittivity of free space. The subscript $i$ denotes the $i$ th relaxation process, $\Delta \varepsilon_{i}=\varepsilon_{s i}-\varepsilon_{\infty i}$ is the dielectric relaxation strength, $\tau_{H N i}$ is the characteristic (Havriliak-Negami) relaxation time, $\alpha_{i}$ and $\beta_{i}$, respectively, are the symmetric

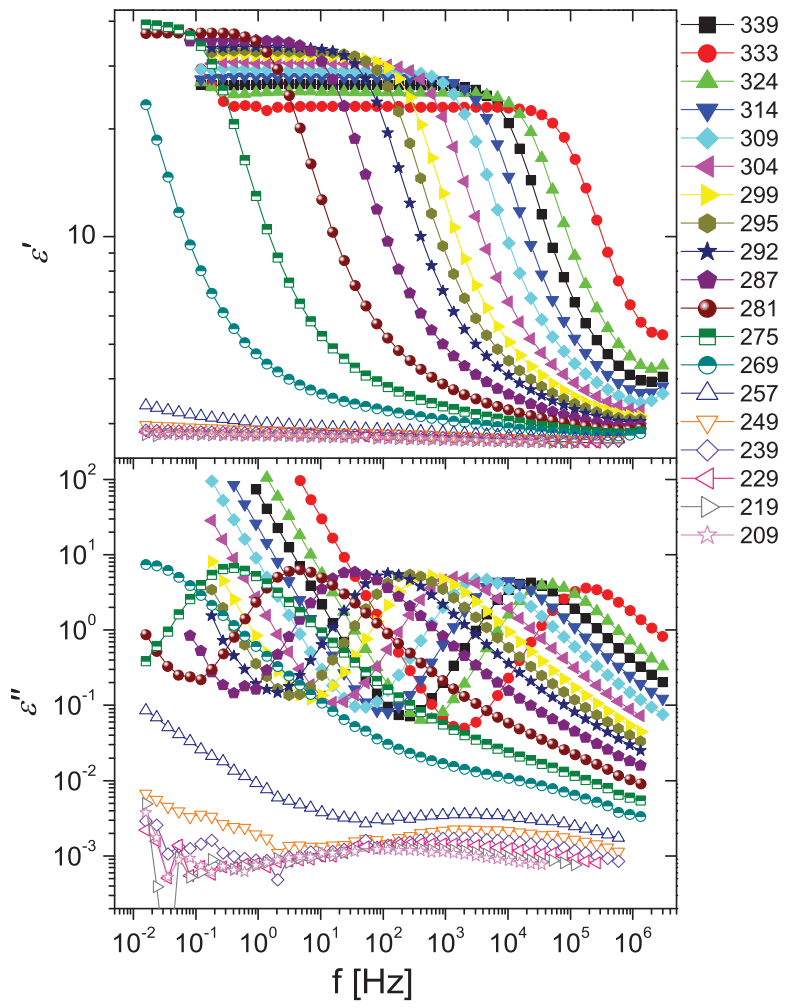

FIG. 3. (a) The dielectric permittivity, $\varepsilon^{\prime}$, and (b) the $\varepsilon^{\prime \prime}$ spectra at a pressure of $1750 \mathrm{MPa}$ at selected temperatures. The temperatures are given in $\mathrm{K}$. and asymmetric shape parameters of the $i$ th process. The term $\sigma_{d c} / \varepsilon_{0} \omega$ is the contribution of dc conductivity to $\varepsilon^{\prime \prime} . \varepsilon_{\infty}$ is the sum of the permittivity contributions from vibrational, ionic, and electronic polarizations. The imaginary part of Eq. (1) was fitted to the experimental $\varepsilon^{\prime \prime}$ spectra using the dielectric fitting program, WinFit, purchased from Novocontrol. Fitting to the real part of permittivity $\varepsilon^{\prime}$ at the atmospheric pressure of $0.1 \mathrm{MPa}$ was carried out to further refine the fitted parameters. For the parameter $\alpha_{i}$ to be unity, $\tau_{H N i}=\tau_{\max i}$. However, if both $\alpha_{i} \neq 1$ and $\beta_{i} \neq 1$, then $f_{m, i}$ is calculated using ${ }^{40}$

$$
f_{m, i}=\left(2 \pi \tau_{H N i}\right)^{-1}\left[\sin \frac{\alpha_{i} \pi}{2+2 \beta_{i}}\right]^{\frac{1}{\alpha_{i}}}\left[\sin \frac{\alpha_{i} \beta_{i} \pi}{2+2 \beta_{i}}\right]^{-\frac{1}{\alpha_{i}}},
$$

where $f_{m, i}$ is the frequency of maximum dielectric loss for the first and the slowest relaxation. $i=\mathrm{II}$ corresponds to the next higher frequency process, and $i=\mathrm{III}$ corresponds to the Johari-Goldstein (JG) process. The latter process will be discussed in a separate section. The relaxation time is found using $\tau_{i}=1 / 2 \pi f_{m, i}$. If $\beta_{i}$ is unity then according to Eq. (2), $\tau_{m, i}=\tau_{H N, i}$ even if $\alpha_{i} \neq 1$.

The dependence of $\tau_{\text {dom }}=\tau_{m}=\tau$ on temperature and pressure can be used to calculate the enthalpy and the volume of activation for both processes by a procedure being outlined here.

When Eyring theory of absolute reaction rates is applied to the dielectric relaxation process/es as was first done by Kauzmann ${ }^{41}$, this leads to the following formula:

$$
\tau=\frac{h}{k T} \exp \left(\frac{\Delta G^{\#}}{R T}\right) .
$$

Here an activated state symbolized as \#, is envisaged in which molecules are free to respond to an external field, $h$ is the Planck's constant, $R$ is the gas constant, and $\Delta G^{\#}$ is the Gibbs free energy of the activation and is expressed in terms of the enthalpy of activation $\Delta H^{\#}$ and the change in the entropy $\Delta S^{\#}$ from the final to the initial state.

$$
\Delta G^{\#}=\Delta H^{\#}-T \Delta S^{\#} .
$$

From equations (3) and (4) we can derive $\Delta H_{\tau}^{\#}$, where the subscript $\tau$ stands for the activation parameters calculated from the data on relaxation time.

$$
\Delta H_{\tau}^{\#}=R\left[\frac{\partial \ln (T \tau)}{\partial(1 / T)}\right]_{P} .
$$

Whalley ${ }^{3}$ has shown that the volume of activation $\Delta V^{\#}$ for the relaxation process is given by

$$
\Delta V_{\tau}^{\#}=R T\left(\frac{\partial \ln \tau}{\partial P}\right)_{T} .
$$

From the linear dependence of $\ln (\tau T)$ with $1 / T$ observed over a limited range of temperatures and from its slope $\Delta H^{\#}$ is found using Eq. (5); $\Delta S^{\#}$ can be found from the intercept of this plot. The Gibbs free energy $\Delta G^{\#}$ can be found using Eq. (4).

Over lower range of pressures, $\ln \tau$ vs. $P$ is linear and from these we can estimate the volume of activation, using 
TABLE I. The distribution of relaxation time parameters $\left(\alpha_{i}\right.$ and $\left.\beta_{i}\right)$ for the two processes I and II at $P \sim 0.1 \mathrm{MPa}$ and for the dominant process at higher pressures. Temperature range of the measurements is given in each case.

\begin{tabular}{lllccc}
\hline \hline $\begin{array}{c}P \\
(\mathrm{MPa})\end{array}$ & $\begin{array}{c}\alpha_{\mathrm{I}} \text { or } \\
\left(\alpha_{\text {dom }}\right)\end{array}$ & $\begin{array}{c}\beta_{\mathrm{I}} \text { or } \\
\left(\beta_{\text {dom }}\right)\end{array}$ & $\alpha_{\mathrm{II}}$ & $\beta_{\mathrm{II}}$ & $\begin{array}{c}\text { Temp. range } \\
(\mathrm{K})\end{array}$ \\
\hline 0.1 & 1.00 & 0.95 & $0.57 \pm 0.09$ & $0.79 \pm 0.05$ & $190-160$ \\
512 & $0.97 \pm 0.03$ & $0.9-0.75$ & $\ldots$ & $\ldots$ & $280-200$ \\
780 & $1.0-0.95$ & $0.82-0.7$ & $\ldots$ & $\ldots$ & $277-200$ \\
1750 & $1.0-0.95$ & $0.55-0.5$ & $\ldots$ & $\ldots$ & $340-270$ \\
\hline \hline
\end{tabular}

Eq. (6). Values of the activation parameters so deduced will be discussed later.

\section{RESULTS AND DISCUSSION}

The distribution parameters of the relaxation processes found by fitting the data to the H-N Eq. (1) are listed in Table I (given for both processes I and II for $P \sim 0.1 \mathrm{MPa}$ ) and for the dominant process at higher pressures.

We find that the parameter $\beta_{\text {dom }}$ at a pressure of $512 \mathrm{MPa}$ decreases from $\sim 0.9$ at $280 \mathrm{~K}$ to $\sim 0.75$ for $200 \mathrm{~K}$. Similarly a reduction in $\beta_{\text {dom }}$ with temperature for a pressure of $780 \mathrm{MPa}$ is observed. At a pressure of $1750 \mathrm{MPa}$, the value of $\beta_{\text {dom }}$ is already low and it varies by $10 \%$ around a mean value of 0.53 for different temperatures.

Figure 4 shows $\varepsilon^{\prime \prime}$ data for pressures of $0.1 \mathrm{MPa}$, $512 \mathrm{MPa}, 780 \mathrm{MPa}$, and $1750 \mathrm{MPa}$ for a temperature of $223 \mathrm{~K}(224 \mathrm{~K}$ in the case of $1750 \mathrm{MPa})$. This figure shows that the dominant $\varepsilon^{\prime \prime}$ peak expressed on the logarithmic scale (belonging to process I), shifts rapidly to lower frequencies, and this continues up to a pressure of $780 \mathrm{MPa}$. The half width

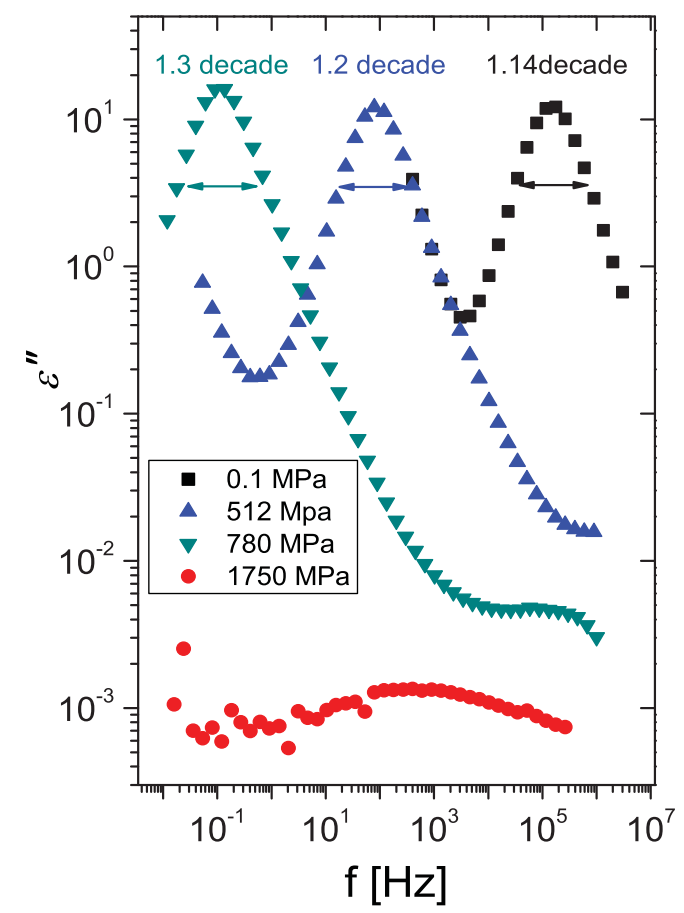

FIG. 4. The dielectric loss, $\varepsilon^{\prime \prime}$ at a temperature of $223 \mathrm{~K}$ and selected pressures of $0.1 \mathrm{MPa}(\boldsymbol{\square}), 512 \mathrm{MPa}(\boldsymbol{\Delta}), 780 \mathrm{MPa}(\boldsymbol{\nabla})$, and $1750 \mathrm{MPa}(\mathbf{\bullet})$. of the $\varepsilon^{\prime \prime}$ peak is seen to increase significantly at higher pressures from its atmospheric value of 1.14 decades. The results of the measurements show that such a change occurs continuously. The peak of $\log \varepsilon^{\prime \prime}$ at a pressure of $1750 \mathrm{MPa}$ has already shifted to much lower frequencies below the window of the experiment. At this temperature and pressure, 5methyl-2-hexanol is already transformed to its glassy state leading $\varepsilon^{\prime \prime}$ to be of the order of $10^{-2}$ in the experimental frequency range of investigations. An almost linear rise in $\log \varepsilon^{\prime \prime}$ due to dc conductivity at lower frequencies for a pressure of $0.1 \mathrm{MPa}$, is seen to decrease with an increase in pressure (not shown in Fig. 4), as expected. Figure 5 shows the dielectric strength $\Delta \varepsilon_{\mathrm{I}}$ calculated for process I (or $\Delta \varepsilon_{d o m}$ for higher pressures) vs. temperature for pressures of $0.1 \mathrm{MPa}, 512 \mathrm{MPa}$, $780 \mathrm{MPa}$, and $1750 \mathrm{MPa}$. This shows that $\Delta \varepsilon_{\mathrm{I}}\left(\right.$ or $\left.\Delta \varepsilon_{\text {dom }}\right)$ decreases slightly with an increase in pressure up to $512 \mathrm{MPa}$ but it then subsequently increases for pressures of $780 \mathrm{MPa}$ and $1750 \mathrm{MPa}$. The temperature range, for which these curves are shown, depends on the pressure-the range decreases with an increase in pressure. At lower temperatures and higher pressures, 5-methyl-2-hexanol goes into its glassy state. For $P=0.1 \mathrm{MPa}, \Delta \varepsilon_{\mathrm{I}}$ and $\Delta \varepsilon_{\mathrm{II}}$ for the two processes are also shown in the inset of Fig. 5. The ratio of $\Delta \varepsilon_{\mathrm{I}} / \Delta \varepsilon_{\mathrm{II}}$ at a pressure of $0.1 \mathrm{MPa}$ varies from 42 at $190 \mathrm{~K}$ to 31 at $160 \mathrm{~K}$. For pressures of $512 \mathrm{MPa}$ and higher, it is not possible to resolve the two processes independently. Figure 6 shows the isochronal superposition with the loss peaks centered at a frequency of

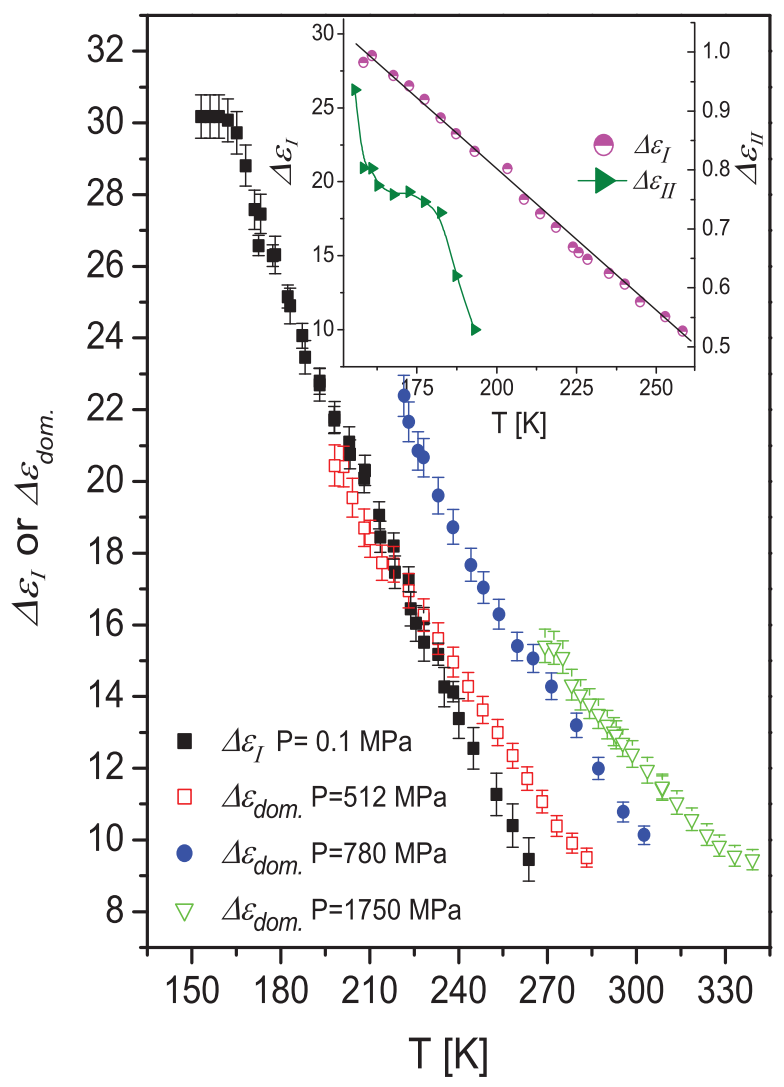

FIG. 5. The dielectric strength as a function of temperature for the selected pressures; $\Delta \varepsilon_{\mathrm{I}}$ for $P=0.1 \mathrm{MPa}(\boldsymbol{\square}) ; \Delta \varepsilon_{d o m}$ for the dominating process for pressures of $P=512 \mathrm{MPa}(\square), 718 \mathrm{MPa}(\circ)$, and $1750 \mathrm{MPa}(\nabla)$. The inset shows $\Delta \varepsilon_{\mathrm{I}}$ and $\Delta \varepsilon_{\mathrm{II}}$ for pure 5-methyl-2 hexanol. 


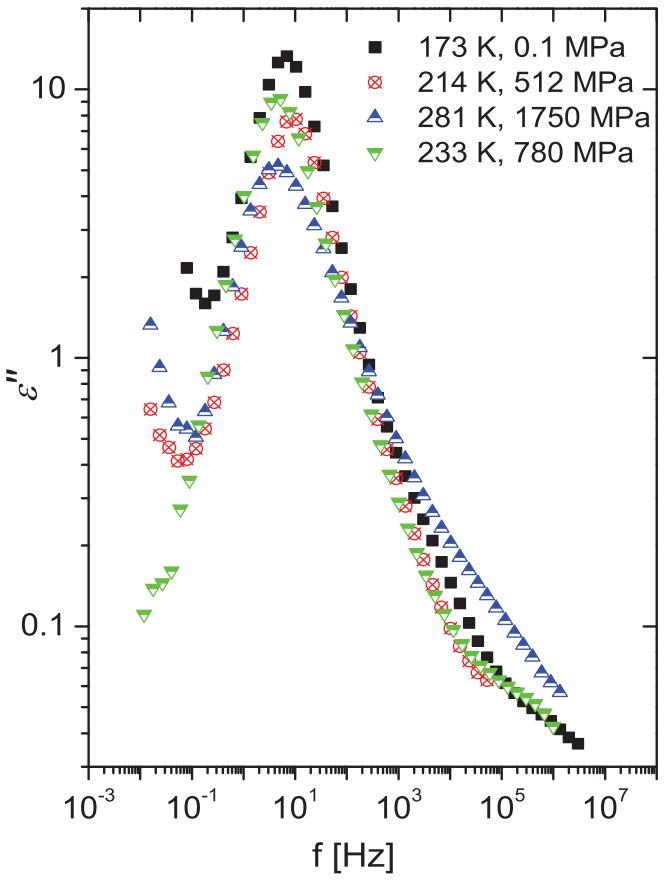

FIG. 6. The dielectric loss for an isochronal temperature-pressure superposition plotted. Under these conditions the relaxation time for the dominating process for the various combinations of $T$ and $P$ are similar. It is clear that the two processes: I and II are resolvable for $P=0.1 \mathrm{MPa}$ merge into a single dominating process for pressures of $P=512 \mathrm{MPa}$ and higher. The high frequency tails in the curves for $780 \mathrm{MPa}$ and $1750 \mathrm{MPa}$ correspond to the JG process that is better resolvable at higher pressures.

$f_{m, i} \sim 8 \mathrm{~Hz}$, where the dominant $\varepsilon^{\prime \prime}$ peak almost coincides for the three sets of temperatures and pressures: $T=173 \mathrm{~K}$, $P=0.1 \mathrm{MPa} ; T=214 \mathrm{~K}, P=512 \mathrm{MPa} ; T=233 \mathrm{~K}$ and $P=780 \mathrm{MPa}$; and $T=281 \mathrm{~K}, P=1750 \mathrm{MPa}$. The height of the loss peak of the dominant peak decreases with elevated pressure. Also the half width of the loss peak is seen to increase significantly at higher pressures from its atmospheric value of 1.14 decades for process I. The curve for the dominant process for a pressure of $1750 \mathrm{MPa}$ is much wider, and this results in an increase in the total dielectric strength for the dominant process, though the height is reduced, as seen in Fig. 6. This becomes even more obvious from the experimental data presented on a master-plot given in Fig. 7 of the $\varepsilon^{\prime \prime}$ spectra for different temperatures for the selected pressure values of $512 \mathrm{MPa}$ and $1750 \mathrm{MPa}$. The isobaric $\varepsilon^{\prime \prime}$ spectra for $P=1750 \mathrm{MPa}$ are superimposed on that calculated using Kohlrausch-Willams-Watts ${ }^{41}$ stretched exponent, $\beta_{\mathrm{KWW}}=0.64$ for the time dependence of the decay in the polarization following the removal of the field; whereas for $P=512 \mathrm{MPa}$, these are superimposed with $\beta_{\mathrm{KWW}} \approx 0.94$. Figure 7 shows that the two processes which are distinct for $P=0.1 \mathrm{MPa}$ appear merged at elevated pressures. This is possibly due to the break-up of the hydrogen bonds and now any reference dipole in the liquid structure is heterogeneous and this in turn leads to a broader distribution of relaxation times. These results appear to be different from those shown by Reiser et al. ${ }^{19}$ for 2-ethyl-1-hexanol, where they show that the peak corresponding to process II increases, whereas the one for process I decreases in amplitude with increase in

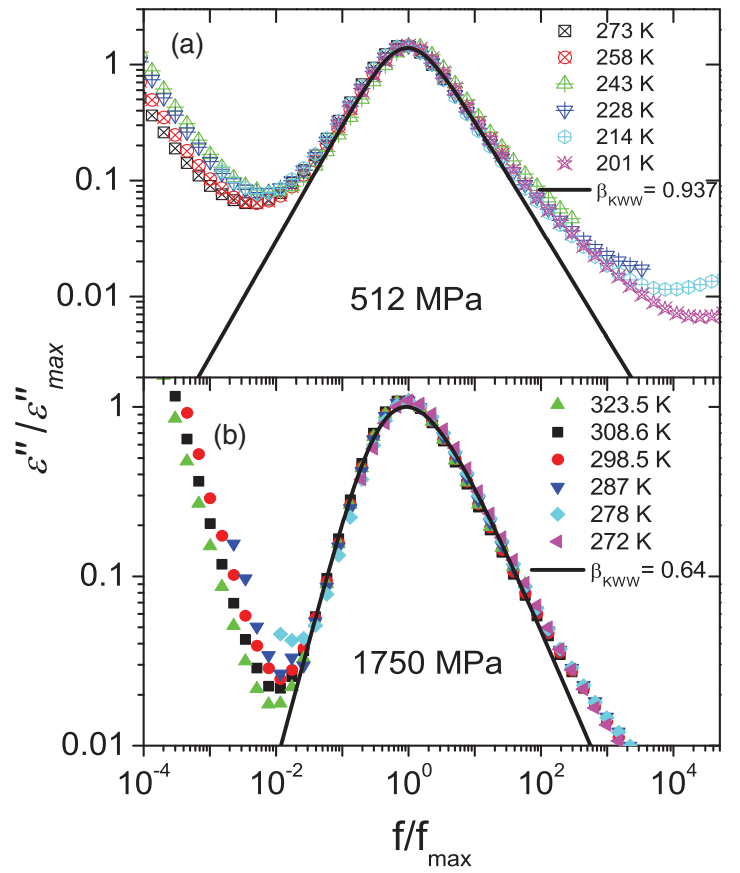

FIG. 7. $\varepsilon^{\prime \prime}$ normalized by $\varepsilon^{\prime \prime} \max$ and frequency, $f$, normalized to the frequency of maximum loss, $f_{\max }$, plotted on a log-log plot for pressures of $512 \mathrm{MPa}$ and $1750 \mathrm{MPa}$. The curves of the dielectric loss are fitted by the Fourier transform of the stretched non-exponential time dependence (KWW

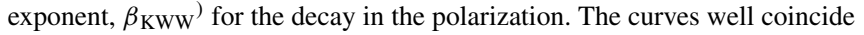
with each other. The high frequency tail is the JG relaxation.

pressure. The two loss peaks in 2-ethyl-1-hexanol are shown apart in the frequency domain for pressures of up to at least $1000 \mathrm{MPa}(10 \mathrm{kbar})$ although both processes markedly approach to each other with densification (see Fig. 5, Ref. 18). This is not, however, seen for 5-methyl 2-hexanol in its pure liquid form. The explanation for the differences in the behaviors of the two alcohols will be given later.

The rise in the conductivity curves for these three sets of temperatures and pressures do coincide better than those observed by Reiser et al. ${ }^{19}$ for 2-ethyl-1-hexanol, again seen in Fig. 7. Figure 8 shows a plot of $T$ vs. $P$ for different selected values of $\Delta \varepsilon_{\text {dom }}$ for the dominant dielectric relaxation process, varying from 10.0 to 13.0. This figure interestingly

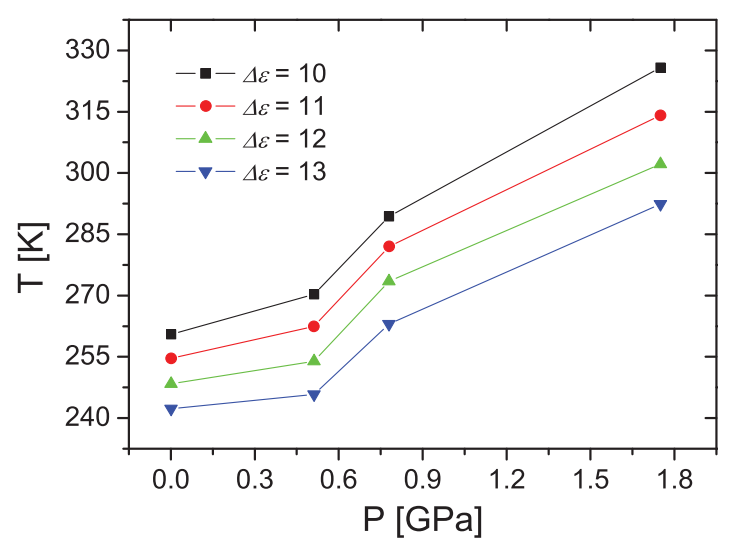

FIG. 8. Temperature and pressure plots for the selected values of the dielectric strengths are plotted. $\Delta \varepsilon_{\mathrm{I}}$ for $P=0.1 \mathrm{MPa}$ but for higher pressures $\Delta \varepsilon_{d o m}$ for the dominating process are considered. 


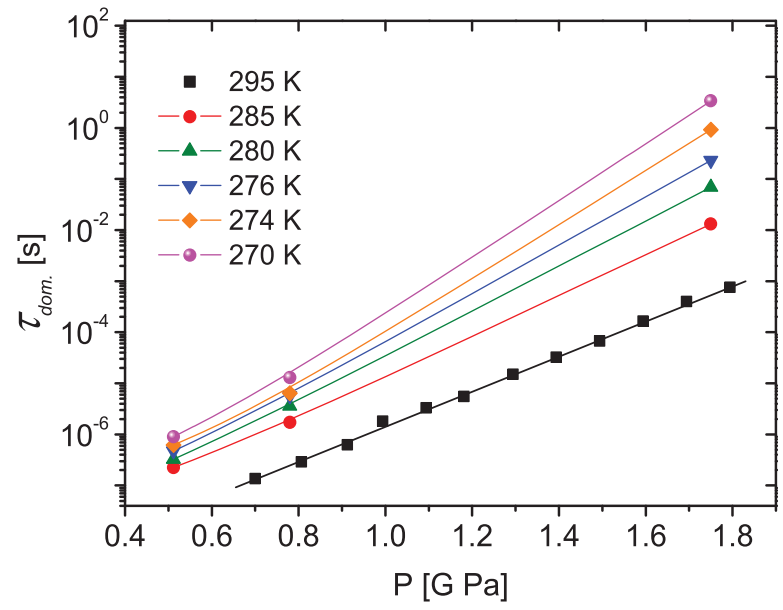

FIG. 9. The relaxation time $\tau_{d o m}$ of the dominating process plotted as a function of pressure for selected temperatures.

shows that for a fixed value of $\Delta \varepsilon_{d o m}$, in the range of the values selected, temperature is increased with elevated pressure, especially above $\sim 500 \mathrm{MPa}$. The set of temperatures, however, for a pressure of $512 \mathrm{MPa}$ are lower compared to a linear extrapolation of these curves alone. This is a consequence of a reduction in $\Delta \varepsilon_{\mathrm{I}}$ caused by an initial rise in pressure. For the same $\Delta \varepsilon_{\mathrm{I}}$, we need to go to lower temperatures. Such a behavior is likely to be a consequence of the break up in the dominant hydrogen bonded structure of 5-methyl-2hexanol in its liquid state with increased pressure as already concluded. Figure 9 shows the change in the relaxation time of the dominant process with increase in the pressure for selected temperatures. This curve reflects an increased relative sensitivity of the relaxation time's dependence on pressure for different temperatures. The relative pressure sensitivity of the relaxation time is found to be much higher for lower temperatures than for higher temperatures. The slope gives a value for the activation volume $\Delta V^{\#}$, defined as the difference in the volume between the activated and non-activated states. A tendency for the divergence in the relaxation time plot vs. pressure is also apparent at higher temperatures. These results extend the trend previously established for heptanols ${ }^{13}$ and octanols ${ }^{5}$ investigated, up to pressures of 350 and $400 \mathrm{MPa}$. For $P<500 \mathrm{MPa}$, the frequency for which $\varepsilon^{\prime \prime}$ peak will occur is beyond the frequency window of the experiment. Normally one would have expected this curve at higher pressures to diverge with pressure but this cannot be visualized due to the absence of the data at lower pressures for these temperatures.

Figure 10 shows isochronal plots of $T$ and $P$ for the various values of the relaxation times of the dominant process $\tau_{\text {dom }}$ varying from $3.3 \mathrm{~s}$ to $10^{-6} \mathrm{~s}$. The behavior on the $T$ and $P$ plane plot is reflected in a continuous change being observed as is expected. The curves on the $T-P$ plot are almost linear at low pressures but then these saturate at higher pressures. These curves show an increased sensitivity of $\tau_{d o m}$ on temperature than on the pressure. Such a trend has also been observed for other hydrogen-bonded systems. ${ }^{5}$ The results in this figure also confirm the previously drawn conclusion that process I does not disappear for any values of $T$ and $P$ in the range of temperatures and pressures investigated. If this pro-

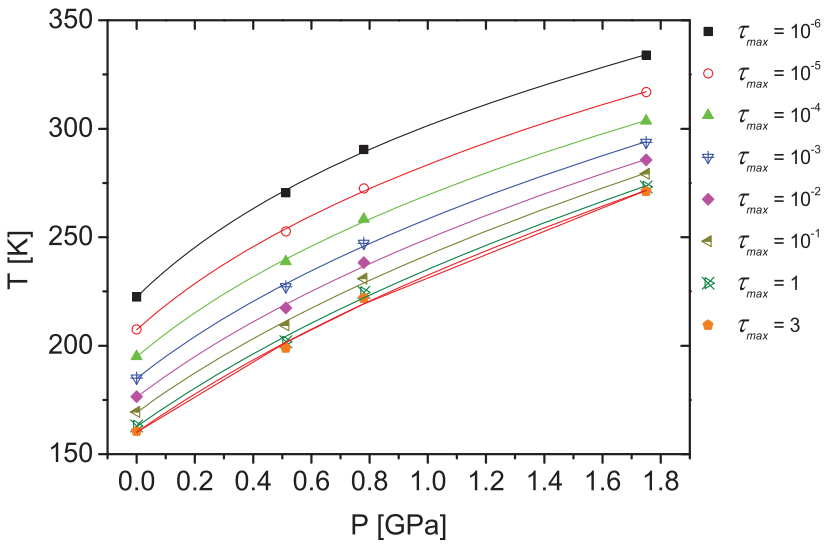

FIG. 10. Temperature vs. pressure plots for isochronal values of the relaxation time (in second). Slopes depend on the relaxation time, indicative of the changes in the structure dependent on the relaxation time. Values of $\mathrm{d} T / \mathrm{d} P$ extrapolated to $P=0.1 \mathrm{MPa}$ are given as follows: $132.3,119.2,112.3,105.6$, $101.2,97.5,95.5$, and 94.1 (all in $\mathrm{K} / \mathrm{GPa}$ ) for relaxation times of $10^{-6}, 10^{-5}$, $10^{-4}, 10^{-3}, 10^{-2}, 10^{-1}, 1$, and $3 \mathrm{~s}$, respectively.

cess were to disappear for some values of $T$ and $P$, and the system were to be controlled by process II, a discontinuity in the curves of Fig. 10 could have easily been observed due to different temperature and pressure sensitivities of the two relaxation processes. The linear parts of the curves are similar to those shown by Johari and Dannhauser ${ }^{5}$ for the five isomers of octanols (Fig. 15, Ref. 5) they investigated; the pressure range in their experiment was, however, limited to $400 \mathrm{MPa}$. These are also similar to the four other isomers of heptanols (see Figs. 5-8, Ref. 13). The data of Fig. 10 are parameterized by using Andersson and Andersson ${ }^{42}$ equation to estimate the value of $\mathrm{d} T / \mathrm{d} P$ for each curve extrapolated to the ambient pressure. The calculated values of $|\mathrm{d} T / \mathrm{d} P|_{\tau}$ markedly increase from $94.1 \mathrm{~K} / \mathrm{GPa}$ for $\tau_{d o m}=3.3 \mathrm{~s}$ to a value of $132.3 \mathrm{~K} / \mathrm{GPa}$ for $\tau_{\text {dom }}=10^{-6} \mathrm{~s}$ with decreasing $\tau_{d o m}$. The values are listed in the figure caption. These differences indicate the changes that occur in the liquid and supercooled state of 5-methyl-2-hexanol for varying values of the relaxation times of the dominant process.

Figure 11 shows a log-linear plot of $\tau_{\mathrm{I}}$ and $\tau_{\mathrm{II}}$ as a function of $1 / T$ for both processes I and II at the atmospheric pressure, whereas the plot of $\tau_{d o m}$ vs. $1 / T$ for pressures of $512 \mathrm{MPa}, 780 \mathrm{MPa}$ and $1750 \mathrm{MPa}$ are shown. Data for $\tau_{\mathrm{II}}$ are shown only for $P \sim 0.1 \mathrm{MPa}$ as $\tau_{\mathrm{II}}$ is sufficiently resolvable for this pressure alone. The increase in the pressure shifts the loss peak to lower frequencies for both processes I and II. The shift is much larger for process II than for process I, such that both processes merge into each other for $P \leq 512 \mathrm{MPa}$ and the $\varepsilon^{\prime \prime}$ curve for the dominant process gets broader. The measurements show that process I does not disappear with increasing pressure and hence it is reasonable to conclude that process II having almost merged in process I is indistinguishable from the latter at pressures of $\sim 512 \mathrm{MPa}$ and above. This behavior contrasts with that for 2-ethyl-1-hexanol. The glass transition temperature of 5-methyl-2-hexanol is $147 \mathrm{~K}$, whereas that of 2-ethyl-1-hexanol is $146 \mathrm{~K}$. These temperatures are rather closer to each other; except that the definition with which these are determined is slightly different. For 


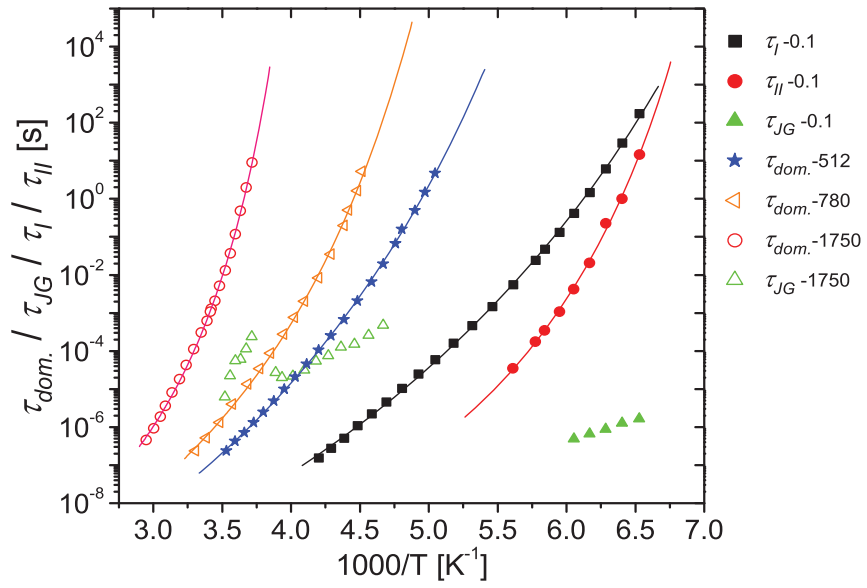

FIG. 11. Relaxation times of processes I and II at $P=0.1 \mathrm{MPa}$ plotted as a function of the inverse absolute temperature. Pressures listed in the figure are in MPa. Relaxation times for the dominating process for $P=512 \mathrm{MPa}$, $780 \mathrm{MPa}$, and $1750 \mathrm{MPa}$ are also plotted as a function of the inverse of absolute temperature. The relaxation time for the JG process is resolved for $P \sim 0.1 \mathrm{MPa}$ and $P=1750 \mathrm{MPa}$.

5-methyl-2-hexanol, we selected $f_{m, \text { II }}=10^{-4} \mathrm{~Hz}$ in our previous works ${ }^{32,35}$ for determining $T_{g}$; whereas $f_{m \text {.II }}=10^{-2}$ $\mathrm{Hz}$ for 2-ethyl-1-hexanol ${ }^{29}$ was used to find $T_{g}$ in the literature. 5-methyl-2-hexanol is an isomer of heptanol, whereas 2-ethyl-1-hexanol is an isomer of octanol (octanols studied by Johari and Goldstein ${ }^{5}$ ). However the data for their viscosities are not known. In one case the hydroxyl group is at the end of the chain (2-ethyl-1-hexanol), the ethyl group is next to it, whereas in the second case (5-methyl-2-hexanol), the hydroxyl group is at position 2 from the end of the chain, and the methyl group is at position 5, with 6 carbon atoms in the chain are present in both cases. For a temperature of $190 \mathrm{~K}$ and $P \sim 0.1 \mathrm{MPa}, \tau_{\mathrm{I}}$ and $\tau_{\mathrm{II}}$ for 5-methyl-2-hexanol are $5 \times 10^{-4} \mathrm{~s}$ and $8 \times 10^{-6} \mathrm{~s}$, respectively, with the ratio of $\tau_{\mathrm{I}} / \tau_{\mathrm{II}}=63$, whereas for 2-ethyl-1-hexanol, these relaxation times are $3 \times 10^{-4} \mathrm{~s}$ and $5 \times 10^{-7} \mathrm{~s}$, respectively, giving a ratio of $\tau_{\mathrm{I}} / \tau_{\mathrm{II}}=600$ in this case. The value of $\tau_{\mathrm{I}}$ for 2-ethyl-1-hexanol is approximately the same as for 5methyl-2-hexanol; however $\tau_{\text {II }}$ for 2-ethyl-1-hexanol is a factor of decade lower than for 5-methyl-2-hexanol. The reason as to why the relaxation process I is of the Debye type in some monohydroxy alcohols is given here. In straight or almost hydrogen-bonded chains formed such as in 2-ethyl-1hexanol, the breaking and reforming of the hydrogen bonds takes longer than the reorientation time of the orientation of the $-\mathrm{OH}$ group dipole moment of the individual molecules around their major molecular axis. Nevertheless the relaxation time for this process is also controlled by the reorientation time of the molecule as well as the cooperative phenomenon of making and breaking the hydrogen bonds here. This process does involve structural fluctuations as the $-\mathrm{OH}$ group has to change its position and this process should therefore contribute to the structural relaxation in spite of the fact that the relaxation process is of the Debye type. The main reason is that the structural fluctuations are faster than the reorientation time of the $-\mathrm{OH}$ group and of the making and breaking of the hydrogen bonds. Since the environments of the refer- ence dipole are homogeneous and these are changing faster than the reorientation time of the molecule then the relaxation time as per Anderson and Ulmann model ${ }^{44}$ is of the Debye type. For the case of 5-methyl-2-hexanol under investigation, the $-\mathrm{OH}$ group as already stated is at position 2 of the carbon chain and the hydrogen-bonded chain is not that straight due to the presence of the methyl group at position 5 (or position 2 from the second end of the chain), the time taken by the $-\mathrm{OH}$ group to change the direction involves the spinning motion about the hydrogen-bonded chain axis which is bent compared to that for 2-ethyl-1-hexanol. The activation energy for process I, $\Delta H^{\#}$, for 5-methyl-2-hexanol $(15.3 \mathrm{kcal} / \mathrm{mol})$ and 2-ethyl-1-hexanol ${ }^{45}(13.6 \mathrm{kcal} / \mathrm{mol})$ are similar to each other. The relaxation time of process II is associated with the rotation of the $-\mathrm{OR}$ group around the $-\mathrm{OH}$ group while the hydrogen bonding may or may not be fixed during this duration of time and involves fluctuations in the dipole moment of 1.29 Debye. This explanation first advanced by Hassion and Cole $^{46}$ is well borne out by the recent results of the two alcohols shown for different temperatures and pressures. Since the alkyl groups are highly disordered, the fluctuation in the structure is easily passed on to the neighboring molecules. The explanation given by Garg and $\mathrm{Smyth}^{47}$ involving the reorientation of the free non-hydrogen bonded molecules is ruled out for the reason that the relaxation time of single molecular orientation is unlikely to be too long and it may also not lead to the Vogel-Fulcher-Tamman ${ }^{48}$ type of temperature dependencies observed for process II with a large steepness index, $m$.

The fact that the dominant process under higher pressures has broader distribution of relaxation times leads to the following interpretation: the increase in the pressure distorts the hydrogen bonds. The making and breaking of the hydrogen bonds with the same neighbors are no longer feasible, and hence the structure around a reference dipole is no longer homogeneous. This heterogeneity in the structure around a reference dipole gives rise to a broader distribution of relaxation times especially at higher pressures. The time duration required for making and breaking of hydrogen bonds in such a distorted structure necessarily includes the re-orientation of dipoles and any motion, whether of the spinning of the $-\mathrm{OH}$ group around the chain axis or the rotation of the $-\mathrm{OR}$ group around the $\mathrm{O}-\mathrm{H}$ bond, is dielectrically indistinguishable. The situation is rather similar to the case for 1-phenyl-1propanol $^{49}$ and 2-phenyl-1-propanol, ${ }^{50}$ where the dominating process in each of these two liquids has broader distribution of relaxation times rather than Debye type process I seen in some monohydroxy alcohols. Though the hydrogen bonding is still extensively present in these two liquids, nevertheless the hydrogen-bonded structure is prevented from being linear in each of these two liquids by the steric hindrance of the bulky phenyl group close to the hydroxyl group. Because of the distorted nature of the hydrogen-bonded chains, the environment around the reference dipole is heterogeneous and consequently the Debye type process (process I) for 1-phenyl1-propanol and 2-phenyl-1-propanol is not observed. These plots of $\log \tau_{\mathrm{I}}$ and $\log \tau_{\mathrm{II}}$ for the two processes as a function of $1 / T$ given in Fig. 11 are fitted to the equation which is a modification of VFT (Ref. 51) and is derived on assumptions 
concerning a change in the specific heat with temperature ${ }^{51}$

$$
\frac{\tau}{\tau_{0}}=\exp \left(\frac{D T_{0}}{T-T_{0}}\right) \text {. }
$$

In equation, $D T_{0}=B, B$ is a parameter of the VFT. The parameters are listed in Table II.

The parameter $D$ is related to the steepness index, $m_{c a l}$, by the following relationship (Eq. (8)).$^{52}$

$$
m_{c a l}=\frac{D T_{0} T_{g}}{\ln 10\left(T_{g}-T_{0}\right)^{2}} .
$$

Here $T_{\mathrm{g}}=T$ for $\tau=10^{2}$. Thus there is an uncertainty in determining $m_{\text {cal }}$ from Eq. (8) because it depends on several fitted parameters. Process III is the JG $\beta$ relaxation process. This is clearly resolvable for the pressures investigated. Plots for $\log \tau_{\text {III }}$ as a function of $1 / T$ for the two pressures of $0.1 \mathrm{MPa}$ and $1750 \mathrm{MPa}$ are also shown in Fig. 11. This will be discussed in Sec. IV D. Figure 12 shows the plot of $\log \tau_{\mathrm{I}}$ and of $\log \tau_{\mathrm{II}}$ versus the inverse of absolute temperature normalized to the glass transition temperature at the atmospheric pressure of $\sim 0.1 \mathrm{MPa}$. The viscosity was first plotted as a function of the inverse normalized temperature by $T_{g}$, by Oldekop, ${ }^{53}$ and was followed by Laughlin and Uhlmann. ${ }^{54}$ Angell, ${ }^{55}$ who first used the plots of the viscosity against $T_{g} / T$, extended the plots later to the dielectric relaxation time versus $T_{g} / T$ similar to that of the Oldekop $\operatorname{plot}^{53}$ for viscosity. Since the data of $\varepsilon^{\prime \prime}$, at pressures of $512 \mathrm{MPa}$ and above are not resolvable; the curves for the relaxation times of the dominant process as a function of $T_{g} / T$ are shown in Fig. 12 for pressures of $512 \mathrm{MPa}, 780 \mathrm{MPa}$, and $1750 \mathrm{MPa}$. The steepness index,

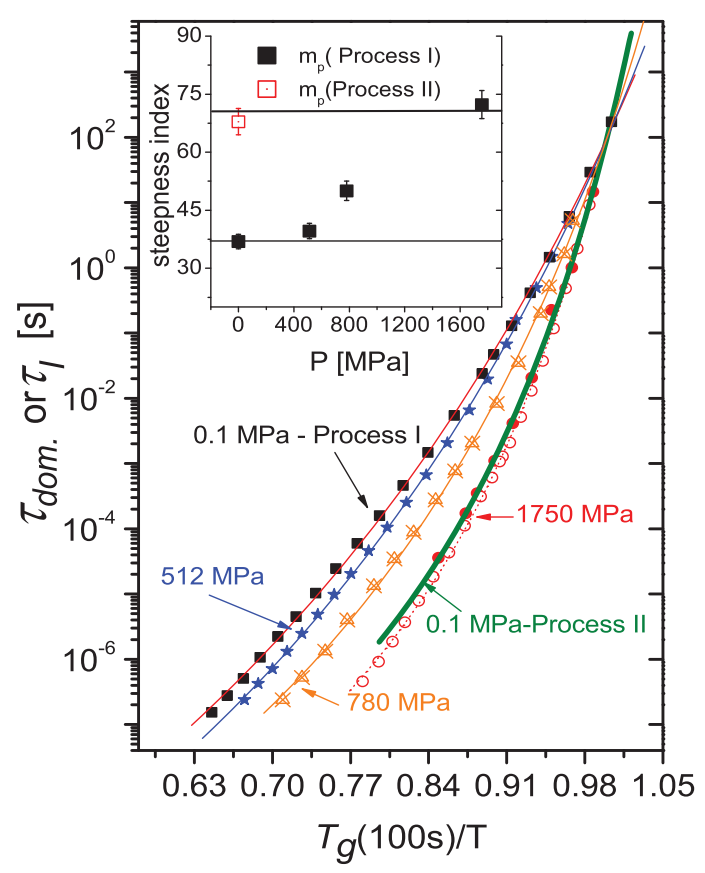

FIG. 12. $\tau_{\mathrm{I}}$ and $\tau_{\text {II }}$ plotted versus inverse of absolute temperature normalized to the glass transition temperature for $P=0.1 \mathrm{MPa}$. For selected higher pressures of $P=512 \mathrm{MPa}, P=718 \mathrm{MPa}$, and $P=1750 \mathrm{MPa}$ : $\log \tau_{d o m}$ for the dominating process is plotted vs. $T_{g} / T$. In the inset, the plot of steepness index, $m$, as a function of pressure is shown. $m_{\text {slope }}$, defined as ${ }^{56}$

$$
m_{\text {slope }}=\left|\mathrm{d}\left(\log \tau_{\text {dom }}\right) / \mathrm{d}\left(T_{g} / T\right)\right|_{T=T g},
$$

is plotted as a function of pressure. It is preferred to calculate $m_{\text {slope }}$ using Eq. (9) as the uncertainty is small. The surprising and the most interesting aspect of the results is that the steepness index (or fragility coined by Angell) increases with pressure from 37 to 72 and for a pressure of $1750 \mathrm{MPa}$, $m_{\text {slope }}$ is almost the same as for process II at the atmospheric pressure. This shows that the dynamic heterogeneity reflected in the steepness index, $m_{\text {slope }}$, as probed by dielectric spectroscopy for process II is the same as that observed for the dominant relaxation process (a merger of processes I and II) for $P=1750 \mathrm{MPa}$. A similar observation has also recently been made on 2-ethyl-1-hexanol. ${ }^{20}$

\section{A. Interpretation of the relaxation time in terms of the barrier height}

Debye $^{57}$ gave two alternative interpretations of the dielectric relaxation time. The most commonly used is based on the Brownian motion in which the relaxation time is linked to kinematic viscosity of the medium. The alternative one is that the relaxation time is determined by the jump over the barrier height. This idea was later developed by Fröhlich ${ }^{21}$ and is now called the Debye-Fröhlich model. According to this model, the relaxation time is associated with the escape rate from the potential well. It is also well known that if the barrier height $\gg \mathrm{kT}$, then the relaxation is of the Debye type..$^{58}$ Even if there were a distribution in the barrier heights, such a variation as percentage of the total barrier height would be small. In liquid crystals, it is known that those modes associated with a large nematic potential: such as the rotation of the dipole around the short axis, are of the Debye type with almost zero distribution of relaxation times. ${ }^{59}$ However, the modes involving the rotation of molecules along their long axes and the precession around the director are usually of the non-Debye type. In ferroelectric liquid crystals, the symmetry breaking mode, known as Goldstone mode, is inevitably of the Debye type whereas other modes such as soft-mode and molecular modes usually are non-Debye. ${ }^{60}$ In view of this model, both Debye and non-Debye processes are equally informative and so long these are thermally activated involve molecular motions, molecular reorientations, and structural fluctuations. It would therefore not be correct to state that only those modes that have distribution of relaxation times are associated with the molecular reorientation and therefore only correspond to the structural relaxation.

\section{B. Structural relaxation}

The parameters, such as the enthalpy and the volume of activation for processes-I and II at $P \sim 0.1 \mathrm{MPa}$, are calculated from the experimental data and are listed in Table III. For the dominant process, these parameters are also calculated for the data at higher pressures. The results are compared with those of the other isomers of heptanols. The enthalpy of activation is calculated from the linear range of $\mathrm{d}[\ln (\tau T)] / \mathrm{d}[1 / T]$. The linearity is observed only at higher tem- 
TABLE II. The values of the VFT parameters $\log \tau=\log \tau_{0}+\left(D^{\prime} \times T_{0}\right) /\left(T-T_{0}\right)$ as defined by Eq. (7); $D^{\prime}=D / \ln 10$.

\begin{tabular}{lcccccc}
\hline \hline$P(\mathrm{MPa})$ & $\log \tau_{0}$ & $D^{\prime}$ & $m_{\text {cal }}$ & $m_{\text {slope }}$ & $\mathrm{T}_{0}(\mathrm{~K})$ & $\mathrm{T}_{\mathrm{g}}(\mathrm{K})$ \\
\hline 0.1 -process I & $-13.30 \pm 0.22$ & $10.82 \pm 0.69$ & $39.5 \pm 2$ & $36.9 \pm 2$ & $90.08 \pm 1.89$ & $151.2 \pm 2$ \\
0.1 -process II & $-11.90 \pm 1.13$ & $3.58 \pm 0.95$ & $59 \pm 5$ & $67.9 \pm 4$ & $120.24 \pm 4.56$ & $153.7 \pm 3$ \\
512 & $-13.53 \pm 0.21$ & $9.94 \pm 0.59$ & $39.7 \pm 2$ & $39.6 \pm 2$ & $116.50 \pm 2.22$ & $191.2 \pm 2$ \\
780 & $-12.64 \pm 0.42$ & $6.05 \pm 0.72$ & $50 \pm 2$ & $50 \pm 2$ & $151.83 \pm 4.23$ & $214.6 \pm 3$ \\
1750 & $-11.93 \pm 0.22$ & $3.32 \pm 0.20$ & $72.2 \pm 2$ & $72.3 \pm 2$ & $213.84 \pm 1.90$ & $264.8 \pm 2$ \\
\hline \hline
\end{tabular}

peratures but the slope increases significantly as temperature approaches $T_{g}$ as expected. The volume of activation is calculated from $\mathrm{d}(\ln \tau) / \mathrm{d} P$, the slope is linear at lower pressures and then saturates with an increase in pressure. We find that these parameters for process I at $P \sim 0.1 \mathrm{MPa}$ are somewhat lower than for process II. Nevertheless these parameters are significantly larger even for process I compared to other liquids. If process I merely reflected making and breaking of hydrogenbonds and no orientation of molecules, one would not normally expect such a large value for the volume of activation. These results lead us to the conclusion that both processes do involve structural fluctuations as well as the orientation of molecules and consequently both processes contribute to the structural relaxation. The fact that process I exhibits Debye process may imply one of the two scenarios: local structure around the reference dipole is homogeneous or that this process cannot probe the dynamic heterogeneity in some of the monohydroxy alcohols such as 5-methyl-2-hexanol. This in no way conflicts with the concept that spatial heterogeneity in general is a prerequisite for glass vitrification. The mode associated with process II is a better probe for dynamic heterogeneity using dielectric spectroscopy. The probe as already stated should not yield the same result as using the experiments on dynamic specific heat.

\section{Dielectric relaxation time and the Kirkwood correlation factor $(g)$}

Table IV lists the dielectric relaxation time of the dominant process (process I for the data at $P \sim 0.1 \mathrm{MPa}$ ), $\tau_{\mathrm{II}}$ and the Kirkwood correlation factor, $g$, at a temperature of $258 \mathrm{~K}$. The calculated values of Kirkwood correlation factor, $g$, and the relaxation times for a selected set of temperature and pressure are also listed. In order to calculate the $g$-factor

TABLE III. $\Delta V^{\#}\left(\mathrm{~cm}^{3} \mathrm{~mol}^{-1}\right)$ and $\Delta H^{\#}\left(\mathrm{kcal} \mathrm{mol}{ }^{-1}\right)$ of 5-methyl-2hexanol.

\begin{tabular}{lccc}
\hline \hline$T$ & $\Delta V^{\#}$ & $\begin{array}{c}\text { Pressure } \\
(\mathrm{MPa})\end{array}$ & $\begin{array}{c}\Delta H^{\#} \\
\left(\mathrm{kcal} \mathrm{mol}^{-1}\right)\end{array}$ \\
\hline 258 & $\left(\mathrm{~cm}^{3} \cdot \mathrm{mol}^{-1}\right)$ & & \\
270 & 30 & 0.1 & $15.3\left(21.6^{\mathrm{a}}\right)$ \\
274 & 28 & 200 & 15.8 \\
276 & 27 & 512 & 23.5 \\
280 & 25 & 780 & 43.5 \\
285 & 23 & & \\
295 & 21 & & \\
\hline \hline
\end{tabular}

${ }^{\mathrm{a}} \Delta H^{\#}$ within parenthesis refers to process-II. at a pressure of $200 \mathrm{MPa}$ and temperature of $258 \mathrm{~K}$ density for 5-methyl-2-hexanol was extrapolated from its close isomer which was already reported by Vij et al. ${ }^{12}$ From a perusal of Table IV and (Table VII, Ref. 13 which includes results calculated from the data for the octanol isomers from Ref. 5), we find there is a definite link in between the relaxation time and the $g$ value of the alcohols. We find higher is the $g$ of an alcohol, lower is the relaxation time and the predominant dynamics is of the Debye type. From Fig. 10, Ref. 13 , we find that this relationship between $\tau$ and $g$ extends to all temperatures and pressures investigated for the isomers of heptanols and octanols. The corresponding relaxation times and $\tau_{\mathrm{I}}$ and $\tau_{\text {II }}$ per unit viscosity for $\mathrm{n}$-heptyl bromide ${ }^{61}$ (similar to 1-heptanol of similar in size to 5-methyl-2-hexanol)) at $328 \mathrm{~K}$ are $56.4 \mathrm{ps}$ and $4.0 \mathrm{ps}$. Viscosities are of similar values especially at higher temperatures. These times are lower by a factor of at least 1000 for the same temperature. This means that hydrogen bonding plays an essential role in determining both values for $\tau_{\mathrm{I}}$ and $\tau_{\mathrm{II}}$. Based on such a link and the perusal of the values of activation volume and the enthalpy calculated so far (Table III), it would not therefore be correct to state that $\tau_{\mathrm{I}}$ is governed by hydrogen bonding alone and $\tau_{\mathrm{II}}$ corresponding to process II, refers to the dynamics of the alkyl chains and the latter process only contributes to the structural relaxation. Based on these experimental findings, it is reasonable to assert that both processes contribute to the structural relaxation. It can also be concluded that mode of process II may probe the dynamic heterogeneity in monohydroxy alcohols.

\section{Johari-Goldstein relaxation process}

Ngai and Paluch discussed the importance of the JG relaxation in glass vitrification and linked it to the $\alpha$ process. ${ }^{62}$ As stated in the introduction, process III is better resolvable at higher pressures and the separation in the frequency domain of the relaxation times for the dominant process/es from the $\mathrm{JG}$ process is much wider. The process is resolvable above $T_{g}$ and is easily measurable both above and below $T_{g}$. The dielectric strength of this process, though not plotted here, is higher for the data at $P=1750 \mathrm{MPa}$ than for $0.1 \mathrm{MPa}$. The interesting aspect of the result is that there is a much bigger change in the slope of the curve (activation energy from a high value to a low value as the sample is cooled below $T_{g}$ ) at temperatures close to $T_{g}$. For $P \sim 0.1 \mathrm{MPa}$, we observed only a small kink in the slope in the plot $f_{m, J G}$ at $T_{g}$ for 5-methyl-2-hexanol. ${ }^{63,64}$ (See Fig. 4, of Ref. 63 and Fig. 5 of Ref. 64). Such a non-monotonic behavior of JG relaxation was observed for epoxide-based thermosetting polymers, ${ }^{65}$ and polypropylene glycol. ${ }^{66}$ The trend in these results also 
TABLE IV. Summary table for the properties of heptanol isomers for $258 \mathrm{~K}$ and $200 \mathrm{MPa}$.

\begin{tabular}{lccccc}
\hline \hline Material & $g$ & $\begin{array}{c}\tau_{\text {dom }} \times 10^{8} \\
(\mathrm{~s})\end{array}$ & $\begin{array}{c}\Delta H^{\#} \\
\left(\mathrm{kcal} \mathrm{mol}^{-1}\right)\end{array}$ & $\begin{array}{c}\Delta V^{\#} \\
\left(\mathrm{~cm}^{3} \mathrm{~mol}^{-1}\right)\end{array}$ & $\begin{array}{c}\text { Temperature } \\
(\mathrm{K})\end{array}$ \\
\hline 5-methyl-2-hexanol & 2.8 & 8 & 15.7 & 30 & 258 \\
1-heptanol & 3.4 & 4.5 & 8.3 & 14 & 258 \\
3-methyl-2-hexanol & 2.0 & 12 & 20 & 31 & 259 \\
2-methyl-2-hexanol & 1.0 & 23 & 25 & 43 & 258 \\
\hline \hline
\end{tabular}

points towards the experimental observations of tripropylene glycol, ${ }^{67}$ though the effect observed for 5-methyl-2-hexanol for a change in the slope of $\log \tau$ vs. $T$ for temperatures close to $T_{g}$ observed here is much bigger. This will be published separately.

\section{CONCLUSIONS}

It is found that increase in pressure initially decreases the dielectric strength of the dominant process for 5-methyl-2hexanol where it broadens the loss curve. These also show that the two relaxation processes, separated from each other on the scale of relaxation times by a factor of 100 at a temperature of $160 \mathrm{~K}$ and 60 at a temperature of $158 \mathrm{~K}$, respectively, merge into each other for $P \geq 500 \mathrm{MPa}$. The dielectric relaxation strength of the merged processes increases with increase in pressure. The value of the steepness index, $m$, for the processes I and II are different for $P \sim 0.1 \mathrm{MPa}$. However value of $m$, for the composite process, which is a merger of the two processes I and II, for $P=1750 \mathrm{MPa}$ is almost the same as for process II at $P \sim 0.1 \mathrm{MPa}$. The results for the volume of activation and enthalpy and the relaxation times show that process I does contribute to the structural relaxation similarly to process II. It would seem that either or both of the two scenarios may be valid: the dynamic heterogeneity is not important for glass vitrification and/or most likely the dynamic heterogeneity is probed by process II. In hydrogen-bonded systems (as well as in water and ice), the dominant process is the making and breaking of hydrogen bonds and this process does incorporate the reorientation of molecules and contributes to viscosity and conductivity of the system. One of the striking observations is that for a temperature of $258 \mathrm{~K}$ and $P \sim 0.1 \mathrm{MPa}$, the extrapolated values of $\tau_{\mathrm{I}}$ and $\tau_{\mathrm{II}}$ for 5-methyl-2-hexanol are 2.2 $\times 10^{-8}$ and $3.5 \times 10^{-12} \mathrm{~s}$, respectively. This would indicate that $\tau_{\mathrm{II}}$ at this temperature at least is governed by the internal rotations of the $\mathrm{C}-\mathrm{O}$ group; the assignment fits in what was proposed by Hassion and Cole. ${ }^{46} \tau_{\mathrm{II}}$ increases remarkably with a reduction in temperature and increment in pressure and so does $\tau_{\mathrm{I}}$. Such a dramatic increase could not have been possible without the hydrogen bonding having a pronounced effect on both of these processes.

\section{ACKNOWLEDGMENTS}

One of the authors (J.K.V.) thanks G. P. Johari and A. Kocot for valuable discussions. M. Paluch is deeply thankful for the financial support received for the research project (Contract No. TEAM/2008-1/6), operated within the Founda- tion for Polish Science Team Programme co-financed by the EU European Regional Development Fund. The work was supported by Ministry of Science and Education (Poland), Grant No. N N202 231737. The research studentship of M. Nagaraj is partially funded by the Trinity College Dublin postgraduate award. We thank T. B. H. McMurry for analyzing the purity of alcohol using NMR.

${ }^{1}$ B. K. P. Scaife, Proc. Phys. Soc. B 68, 790 (1955).

${ }^{2}$ G. E. McDuffe and M. V. Kelly, J. Chem. Phys. 41, 2666 (1964).

${ }^{3}$ E. Whalley, Adv. High Press. Res. 1, 143 (1966).

${ }^{4}$ W. G. S. Scaife, Annual Report of the Conference on Electrical Insulation and Dielectric Phenomenon (National Academic Science, Washington, DC, 1967), p. 70.

${ }^{5}$ G. P. Johari and W. Dannhauser, J. Chem. Phys. 50, 1862 (1969).

${ }^{6}$ T. Chen, W. Dannhauser, and G. P. Johari, J. Chem. Phys. 50, 2046 (1969).

${ }^{7}$ Yu. A. Atanov and M. I. Shakhparanov, Russ. J. Phys. Chem. 43, 958 (1969).

${ }^{8}$ W. G. S. Scaife, J. Phys. A: Gen. Phys. 4, 413 (1969).

${ }^{9}$ G. P. Johari and E. Whalley, Faraday Symp. Chem. Soc. 6, 23 (1972).

${ }^{10}$ W. G. S. Scaife, J. Phys. D: Appl. Phys. 9, 1489 (1976).

${ }^{11}$ J. K. Vij and W. G. S. Scaife, J. Chem. Phys. 64, 2226 (1976).

${ }^{12}$ J. K. Vij, W. G. Scaife, and J. H. Calderwood, J. Phys. D: Appl. Phys. 11, 545 (1978) (In Table 1, row 3, 2-methyl-2-hexanol should be read as 3-methyl-2-hexanol).

${ }^{13}$ J. K. Vij, W. G Scaife, and J. H. Calderwood, J. Phys. D: Appl. Phys. 14, 733 (1981) (In Table 5 of this reference, 3-methyl-2-heptanol and 2-methyl2-heptanol should be read as 3-methyl-2-hexanol and 2-methyl-2-hexanol).

${ }^{14}$ C. M. Roland, S. Hensel-Bielowka, M. Paluch, and R. Casalini, Rep. Prog. Phys. 68, 1405 (2005).

${ }^{15}$ F. Kremer and A. Schönhals, Broadband Dielectric Spectroscopy (Springer, New York, 2003), Chaps. 1 and 2.

${ }^{16}$ P. W. Bridgman, Proc. Am. Acad. Arts Sci. 61, 57 (1926).

${ }^{17}$ W. E. Danforth, Phys. Rev. 38, 1224 (1931).

${ }^{18}$ D. Fragiadakis, C. M. Roland, and R. Casalini, J. Chem. Phys. 132, 144505 (2010).

${ }^{19}$ A. Reiser, G. Kasper, C. Gainaru, and R. Böhmer, J. Chem. Phys. 132, 181101 (2010).

${ }^{20}$ S. Pawlus, M. Paluch, and M. Dzida, Phys. Chem. Lett. 1, 3249 (2010).

${ }^{21} \mathrm{H}$. Fröhlich, Theory of Dielectrics, 2nd ed. (Clarendon, Oxford, 1958).

${ }^{22}$ W. Dannhauser, J. Chem. Phys. 48, 1918 (1968).

${ }^{23}$ B. Singh and J. K. Vij, Bull. Chem. Soc. Jpn. 49, 1824 (1976)

${ }^{24}$ G. P. Johari and M. Goldstein, J. Chem. Phys. 53, 2372 (1970).

${ }^{25}$ D. W. Davidson and R. H. Cole, J. Chem. Phys. 19, 1484 (1951).

${ }^{26}$ W. Dannhauser, J. Chem. Phys. 48, 1918 (1968).

${ }^{27}$ S. J. Bass, W. I. Nathan, R M. Meighan, and R. H. Cole, J. Phys. Chem. 68, 509 (1964).

${ }^{28}$ W. Dannhauser and G. P. Johari, Can. J. Chem. 46, 3143 (1968).

${ }^{29}$ H. Huth, L.-M. Wang, G. Schick, and R. Richert, J. Chem. Phys. 126, 104503 (2007).

${ }^{30}$ B. Jacobsen, C. Maggi, T. Christenen, and J. C. Dyre, J. Chem. Phys. 129, 184502 (2008).

${ }^{31}$ C. Gainaru, R. Meier, S. Schildmann, C. Ledrle, W. Hiller, E. A. Rössler, and R. Böhmer, Phys. Rev. Lett. 105, 258303 (2010).

${ }^{32}$ O. E. Kalinovskaya and J. K. Vij, J. Chem. Phys. 112, 3262 (2000).

${ }^{33}$ G. Power, M. Nagaraj, J. K. Vij, and G. P. Johari, J. Chem. Phys. 134, 044525 (2011).

${ }^{34}$ G. Power, J. K. Vij, and G. P. Johari, J. Chem. Phys 126, 034512 (2007).

${ }^{35}$ G. Power, O. E. Panarina, and J. K. Vij, J. Phys. Condens. Matter 19, 506208 (2007). 
${ }^{36}$ G. Power, G. P. Johari, and J. K. Vij, J. Chem. Phys. 116, 4192 (2002).

${ }^{37}$ G. Power, J. K. Vij, and G. P. Johari, J. Phys. Chem. B 111, 11201 (2007).

${ }^{38}$ E. Tombari, C. Ferrari, G. Salvetti, and G. P. Johari, J. Chem. Phys. 130, 124505 (2009).

${ }^{39}$ S. Havriliak Jr., and S. Negami, Polymer 8, 161 (1967).

${ }^{40}$ C. Hansen, F. Stickel, T. Berger, R. Richert, and E. W. Fischer, J. Chem. Phys. 17, 1086 (1997).

${ }^{41}$ W. Kauzmann, Rev. Mod. Phys. 14, 12 (1942).

${ }^{42}$ G. Williams and D. C. Watts, Trans. Faraday Soc. 66, 80 (1970).

${ }^{43}$ S. P. Andersson and O. Andersson, Macromolecules 31, 2999 (1998).

${ }^{44}$ J. E. Anderson, and R. Ullman, J. Chem. Phys. 47, 2178 (1967).

${ }^{45}$ R. G. Bennett and J. H. Calderwood, Proceedings of 4th International Meeting on Molecular Spectroscopy, Bologna, 1959, Vol. 3, p. 1182 (1962).

${ }^{46}$ F. X. Hassion and R. H. Cole, J. Chem. Phys. 23, 1756 (1955).

${ }^{47}$ S. K. Garg and C. P. Smyth, J. Phys. Chem. 69, 1294 (1965).

${ }^{48}$ H. Vogel, Phys. Z. 22, 645 (1921); G. S. Fulcher, J. Am. Ceram. Soc. 8, 339 (1923); G. Tammann and W. Z. Hesse, Z. Anorg. Allg. Chem. 156, 245 (1926).

${ }^{49}$ G. P. Johari, O. E. Kalinovskaya, and J. K. Vij, J. Chem. Phys. 114, 4634 (2001).

${ }^{50}$ O. E. Kalinovskaya, J. K. Vij, and G. P. Johari, J. Phys. Chem. A 105, 5061 (2001).

${ }^{51}$ I. M. Hodge, J. Am. Ceram. Soc. 91, 766 (2008).
${ }^{52}$ R. Böhmer, K. L. Ngai, C. A. Angell, and D. J. Plazek, J. Chem. Phys. 99, 4201 (1993).

${ }^{53}$ W. Oldekop, Glastech. Ber. 30, 8 (1957).

${ }^{54}$ W. T. Laughlin and D. R. Uhlmann, J. Phys. Chem. 78, 2317 (1972).

${ }^{55}$ C. A. Angell, J. Non-Cryst. Solids 131-133, 13 (1991).

${ }^{56}$ D. J. Plazek and K. L. Ngai, Macromolecules 24, 1222 (1991).

${ }^{57}$ P. Debye, Polar Molecules (Chemical Catalog, New York, 1929, reprinted by Dover, New York).

${ }^{58}$ F. Marchesoni, J. K. Vij, and W. T. Coffey, Z. Phys. B 61, 357 (1985).

${ }^{59}$ K. Merkel, A. Kocot, J. K. Vij, G. H. Mehl, and T. Meyer, Phys. Rev. E 73, 051702 (2006).

${ }^{60}$ H. Xu, J. K. Vij, A. Rappaport, and N. A. Clark, Phys. Rev. Lett. 79, 249 (1997).

${ }^{61}$ K. Higasi, Dielectric Relaxation and Molecular Structure, Research Monograph No. 9, Research Institute of Applied Electricity, Hokkaido University, Sapporo, Japan, 1961.

${ }^{62}$ K. L. Ngai and M. Paluch, J. Chem. Phys. 120, 857 (2004).

${ }^{63}$ G. P. Johari, G. Power, and J. K. Vij, J. Chem. Phys. 117, 1714 (2002).

${ }^{64}$ J. K. Vij and G. Power, J. Non-Cryst. Solids 357, 783 (2011).

${ }^{65}$ S. Corezzi, M. Beiner, H. Huth, K. Schröter, S. Capaccioli, R. Casalini, D. Fioretto, and E. Donth, J. Chem. Phys. 117, 2435 (2002).

${ }^{66}$ K. Grzybowska, A. Grzybowski, J. Ziolo, M. Paluch, and S. Capaccioli, J. Chem. Phys. 125, 044904 (2006).

${ }^{67}$ M. Paluch, S. Pawlus, S. Hansel-Bielowka, K. Kaminski, T. Psurek, S. J. Rzoska, J. Ziolo, and C. M. Roland, Phys. Rev. B 72, 224205 (2005). 